UDC 622.765(075), 625.75.4

SCOPUS CODE 2201

https://doi.org/10.36073/1512-0996-2020-1-94-100

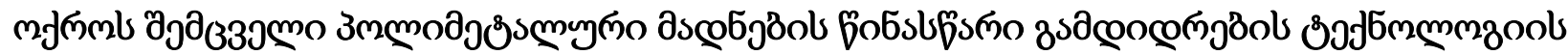
33ल03s

\begin{tabular}{|c|c|}
\hline 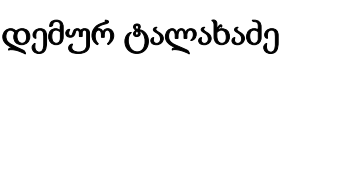 & 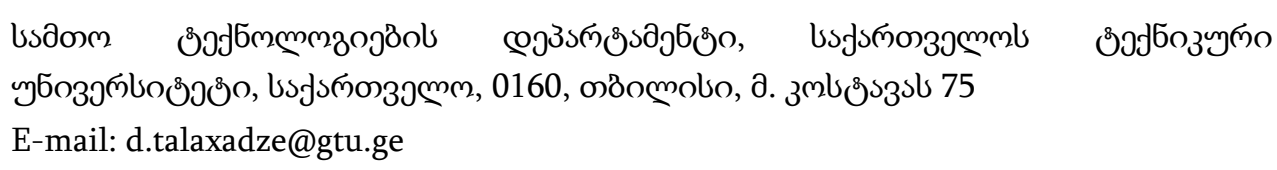 \\
\hline 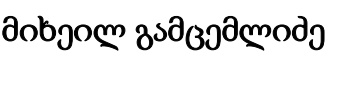 & 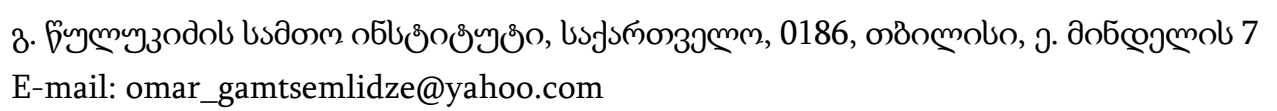 \\
\hline ๓моб јбззэщо & 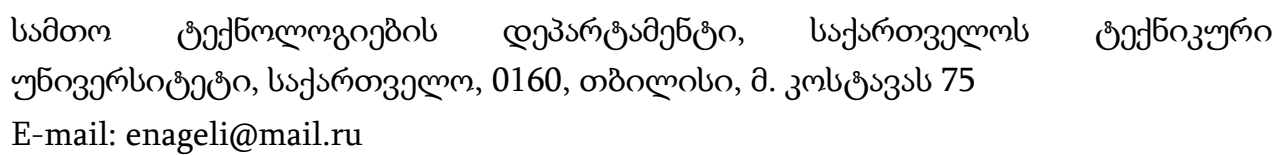 \\
\hline
\end{tabular}

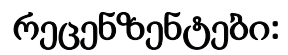

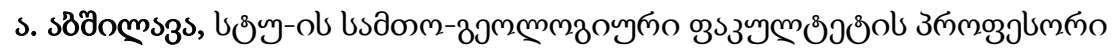

E-mail: a.abshilava@gtu.ge

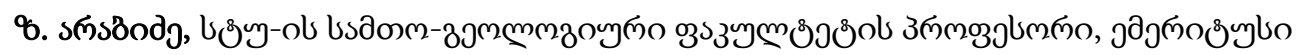

E-mail: z.arabidze@gtu.ge

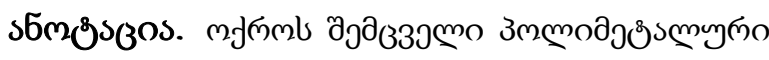

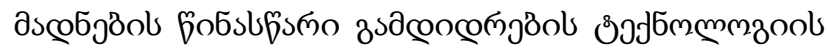

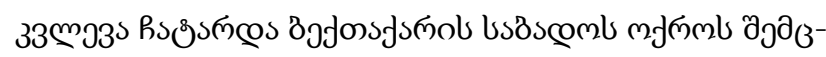

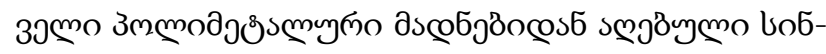

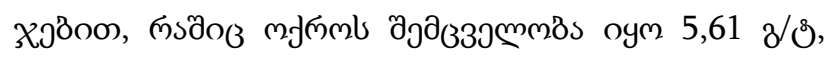

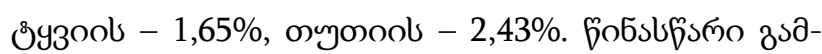

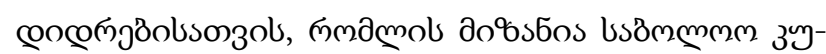

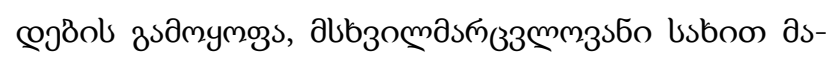

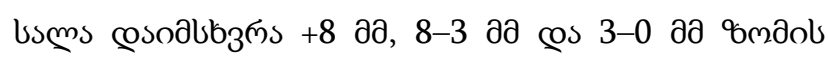

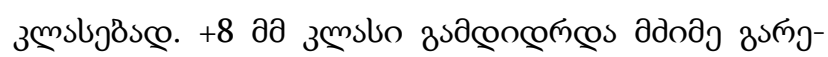

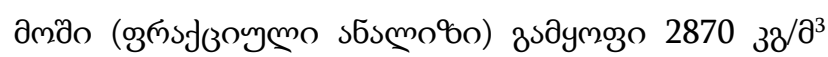

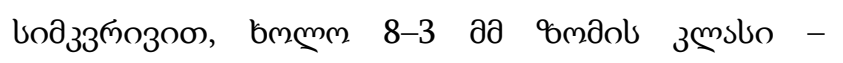

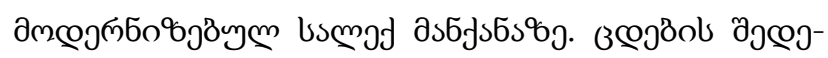

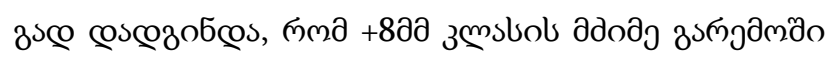

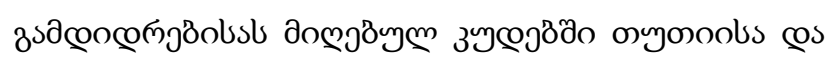

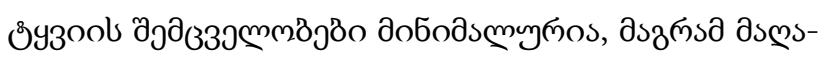

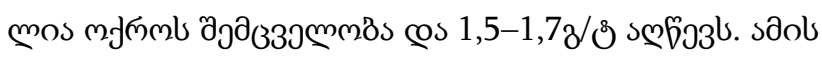

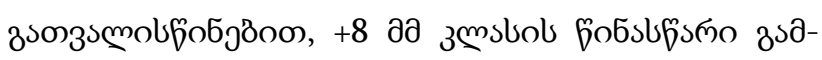

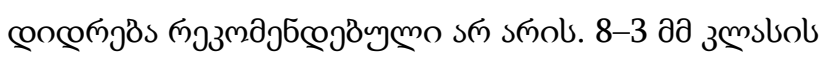

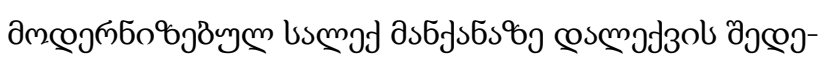

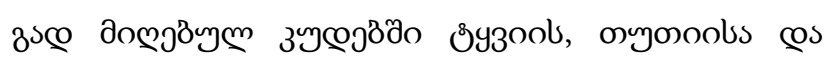

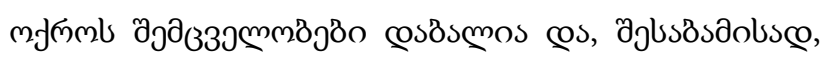
$\mathrm{Pb}=0,24 \%, \quad \mathrm{Zn}=0,32 \%$ cos $\mathrm{Au}=0,4 \quad$ z/§

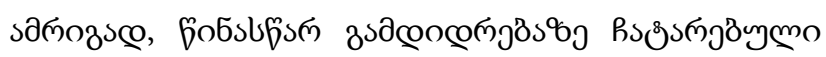

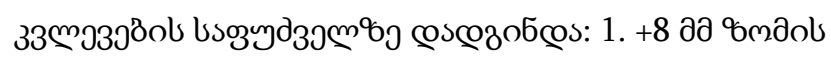




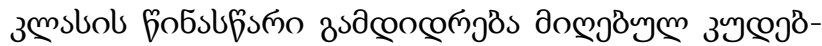

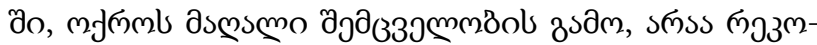

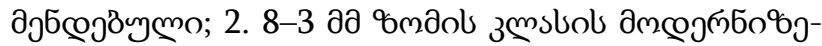

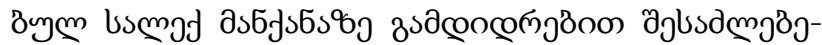

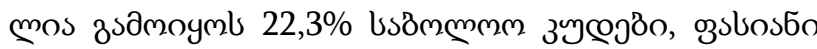

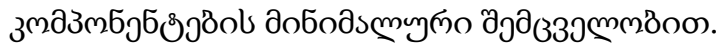

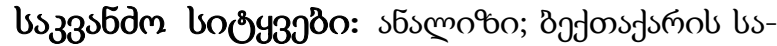

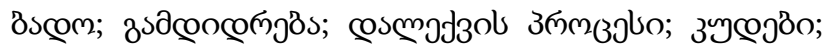

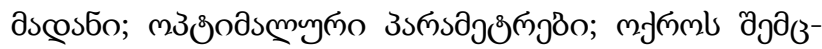
зэмо.

\section{әjиsзsмо}

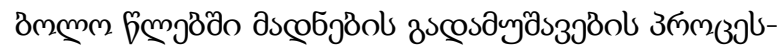

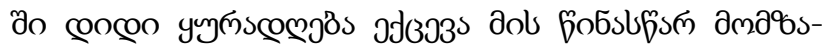

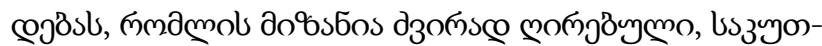

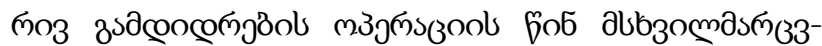

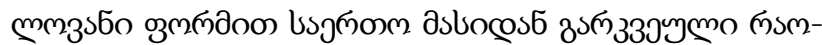

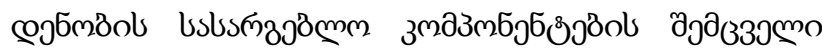

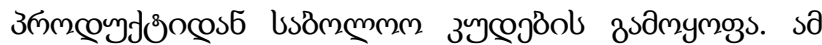

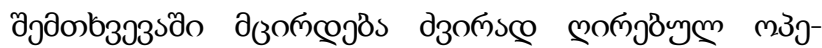

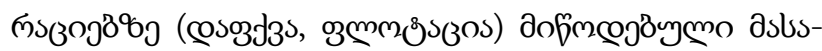

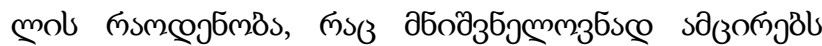

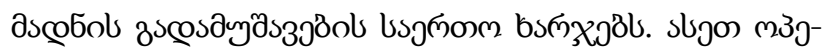

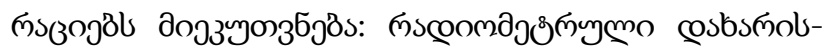

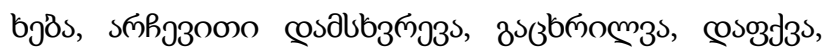

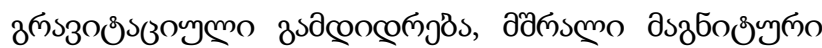
bgzsms zоos.

\section{domooscen 6sformo}

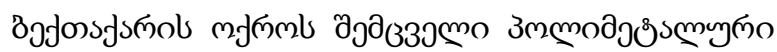

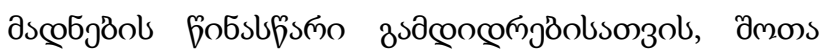

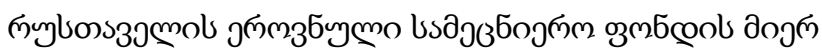

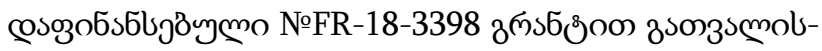

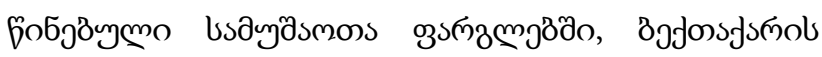

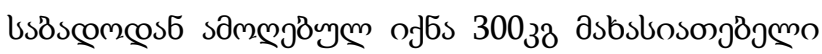

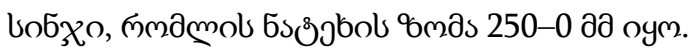

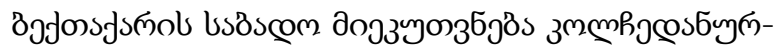

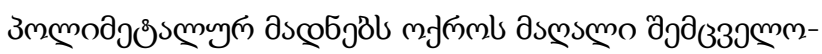

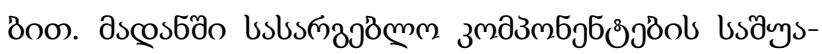

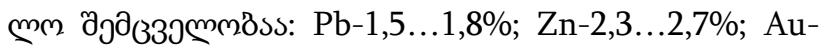

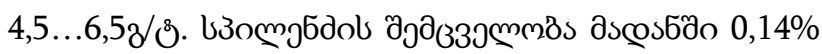

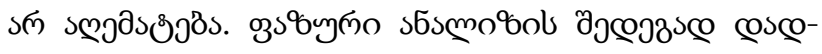

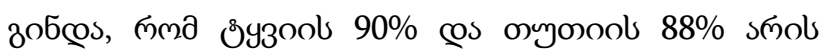

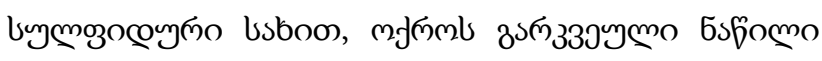

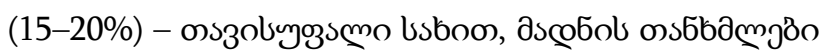

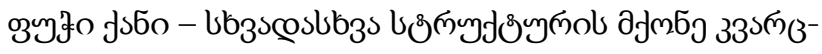

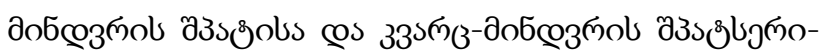

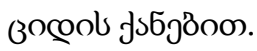

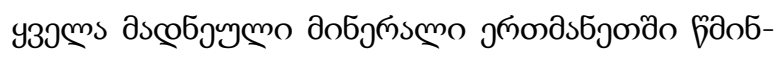

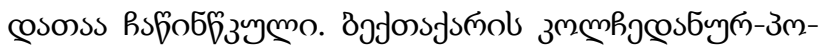

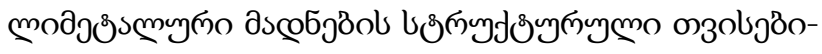

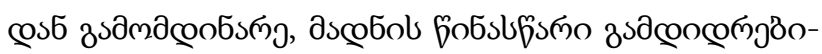

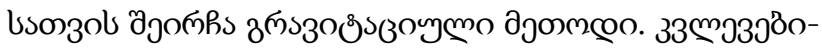

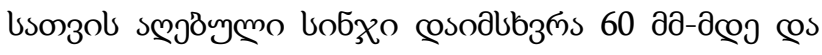

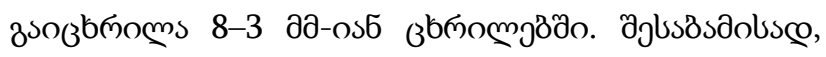

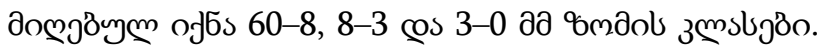

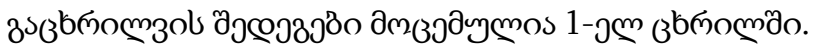




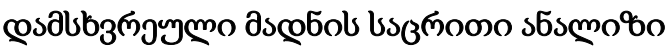

\begin{tabular}{|c|c|c|c|c|c|c|c|}
\hline \multirow{2}{*}{$\begin{array}{c}\text { зmsbjöo } \\
\text { да }\end{array}$} & \multirow{2}{*}{ 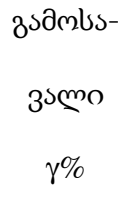 } & \multicolumn{3}{|c|}{ 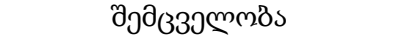 } & \multicolumn{3}{|c|}{ sдмзипозь, \% } \\
\hline & & $\mathrm{Pb}, \%$ & $\mathrm{Zn}, \%$ & $\begin{array}{l}\mathrm{Au}, \\
3^{(m / \delta 5}\end{array}$ & $\mathrm{Pb}$ & Zn & $\mathrm{Au}$ \\
\hline $60-8$ & 51,7 & 1.78 & 2.47 & 5.61 & 48.28 & 46.23 & 47.78 \\
\hline $8-3$ & 35,2 & 1.89 & 2.83 & 5.92 & 34.92 & 36.07 & 34.40 \\
\hline $3-0$ & 13,1 & 2.45 & 3.73 & 8.26 & 16.80 & 17.70 & 17.82 \\
\hline $60-0$ & 100 & 1.9 & 2.76 & 6.07 & 100 & 100 & 100 \\
\hline
\end{tabular}

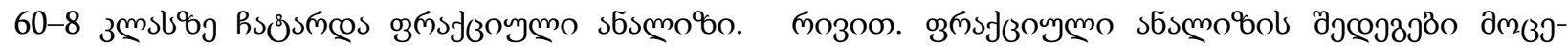

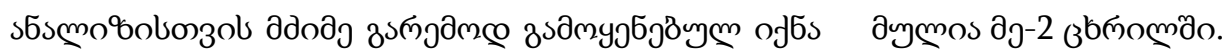

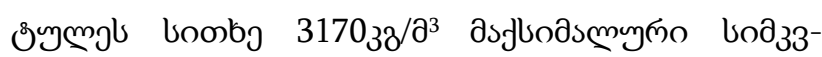

(3) nоmo 2

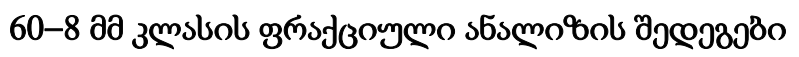

\begin{tabular}{|c|c|c|c|c|c|c|c|c|}
\hline 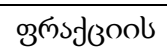 & \multicolumn{2}{|c|}{ 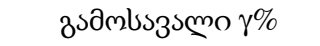 } & \multicolumn{3}{|c|}{ 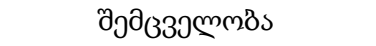 } & \multicolumn{3}{|c|}{ ১дмзљпуоз, \% } \\
\hline 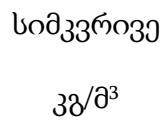 & mӟбпьв. & usগோом & $\mathrm{Pb}, \%$ & $\mathrm{Zn}, \%$ & $\begin{array}{l}\mathrm{Au}, \\
\beta^{(n / 86} 6\end{array}$ & $\mathrm{~Pb}$ & $\mathrm{Zn}$ & $\mathrm{Au}$ \\
\hline+3170 & 5,2 & 2,69 & 8,96 & 10,74 & 18,30 & 26,17 & 22,62 & 16,96 \\
\hline $3170-2980$ & 9,3 & 4,81 & 1,93 & 8,16 & 12,55 & 26,1 & 30,72 & 20,81 \\
\hline $2980-2820$ & 35,7 & 18,46 & 3,76 & 2,73 & 7,1 & 38,71 & 39,52 & 45,2 \\
\hline $2820-2710$ & 20,2 & 10,44 & 0,37 & 0,42 & 2,21 & 4,2 & 3,43 & 7,96 \\
\hline-2710 & 29,6 & 15,3 & 0,29 & 0,31 & 1,72 & 4,82 & 3,71 & 9,07 \\
\hline bymm & 100 & 51,7 & 1,78 & 2,47 & 5,31 & 100 & 100 & 100 \\
\hline
\end{tabular}

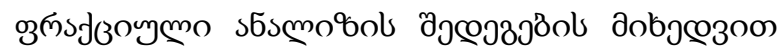

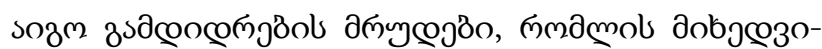

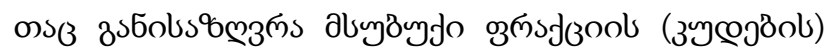

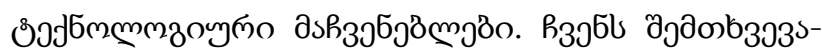

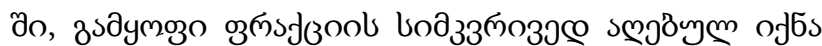

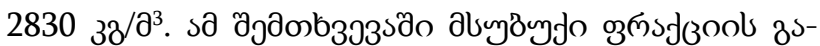

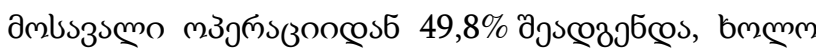

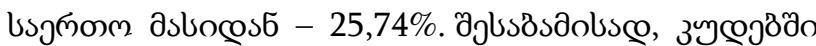

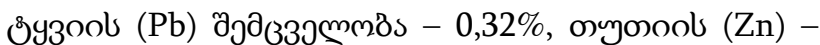

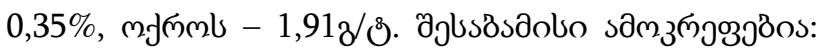




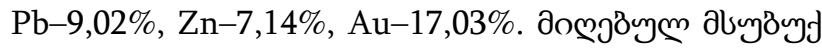

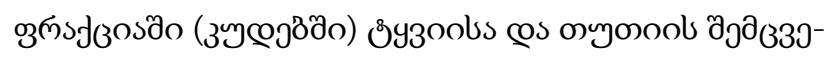

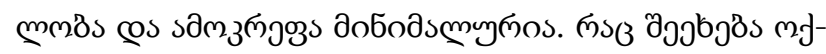

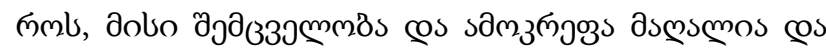

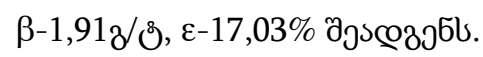

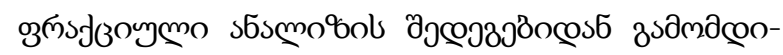

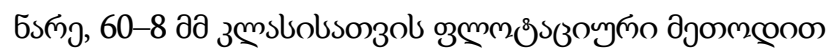

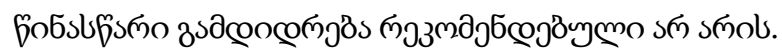

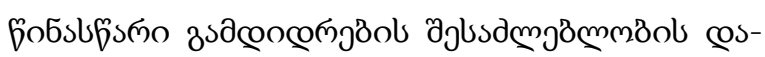

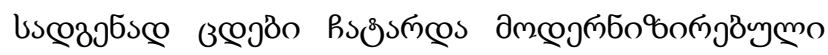

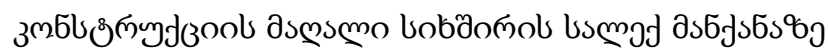

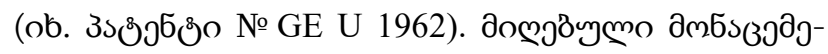

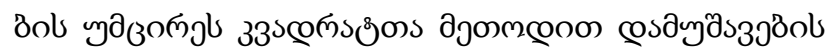

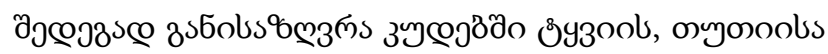

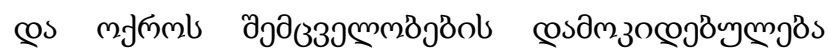

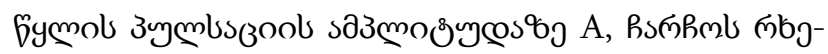

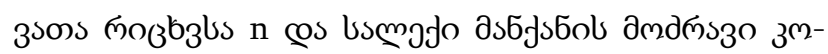

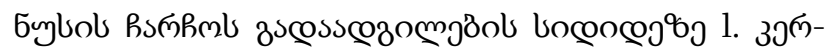

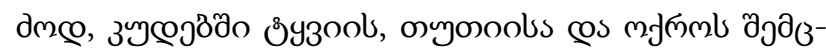

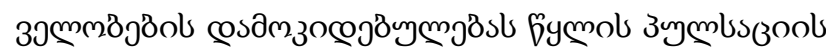

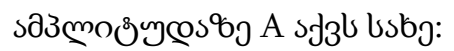

$$
\begin{aligned}
& \theta_{\mathrm{Pb}}=0.022 \mathrm{~A}^{2}-0.701 \mathrm{~A}+6.212, \\
& \theta_{\mathrm{Zn}}=0.038 \mathrm{~A}^{2}-1.192 \mathrm{~A}+10.06, \\
& \theta_{\mathrm{Au}}=0.034 \mathrm{~A}^{2}-1.11 \mathrm{~A}+9.363 .
\end{aligned}
$$

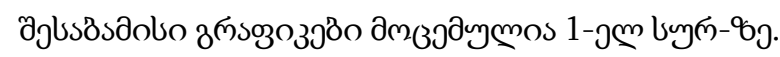

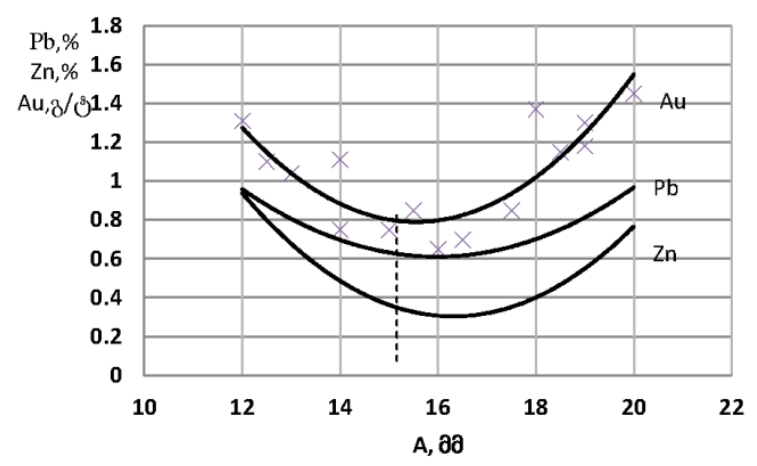

vegn. 1

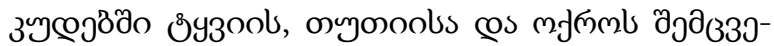

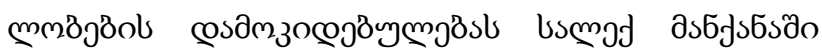

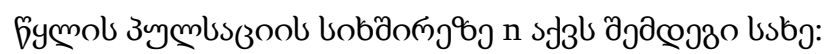

$$
\begin{aligned}
& \theta_{\mathrm{Pb}}=3 \cdot 10^{-5} \mathrm{n}^{2}-0.023 \mathrm{n}+5.649, \\
& \theta_{\mathrm{Zn}}=5 \cdot 10^{-5} \mathrm{n}^{2}-0.046 \mathrm{n}+10.24, \\
& \theta_{\mathrm{Au}}=8 \cdot 10^{-5} \mathrm{n}^{2}-0.066 \mathrm{n}+14.57 .
\end{aligned}
$$

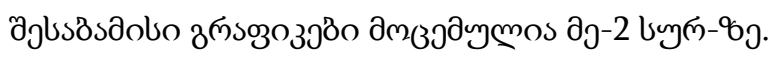

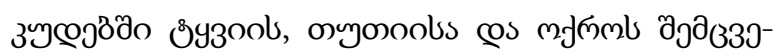

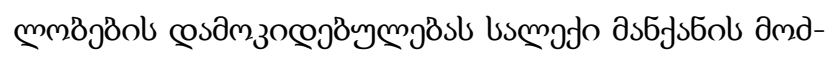

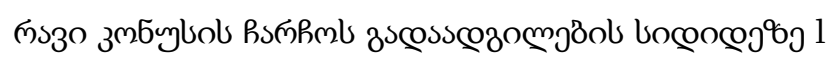

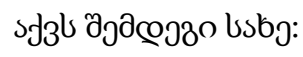

$$
\begin{aligned}
& \theta_{\mathrm{Pb}}=0.015 \cdot 1^{2}-0.307 \cdot 1+2.163, \\
& \theta_{\mathrm{Zn}}=0.033 \cdot 1^{2}-0.648 \cdot 1+3.505, \\
& \theta_{\mathrm{Au}}=0.05 \cdot 1^{2}-0.898 \cdot 1+4.843 .
\end{aligned}
$$

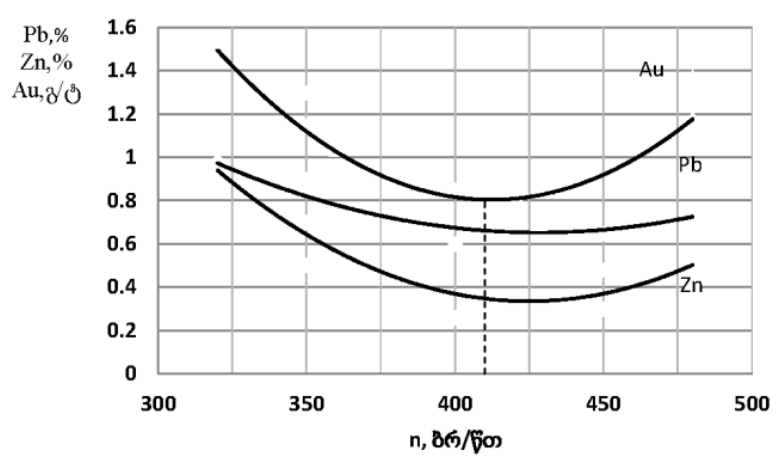

byñ. 2

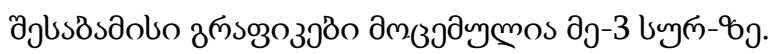

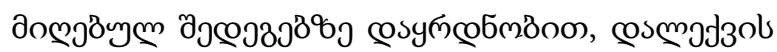

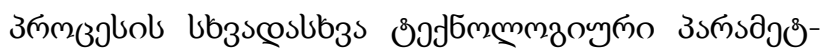

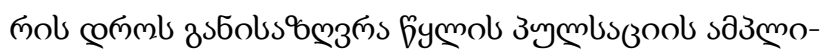

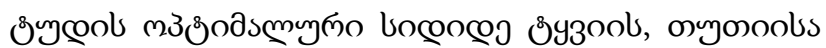

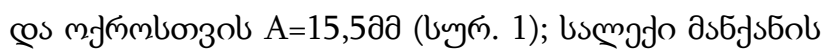

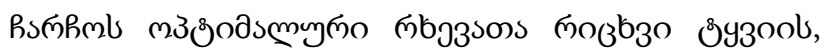

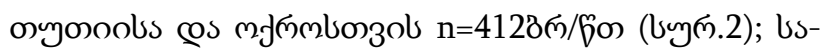

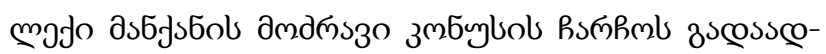

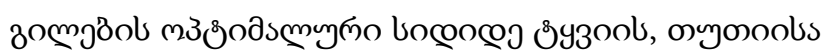

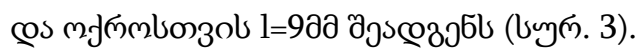




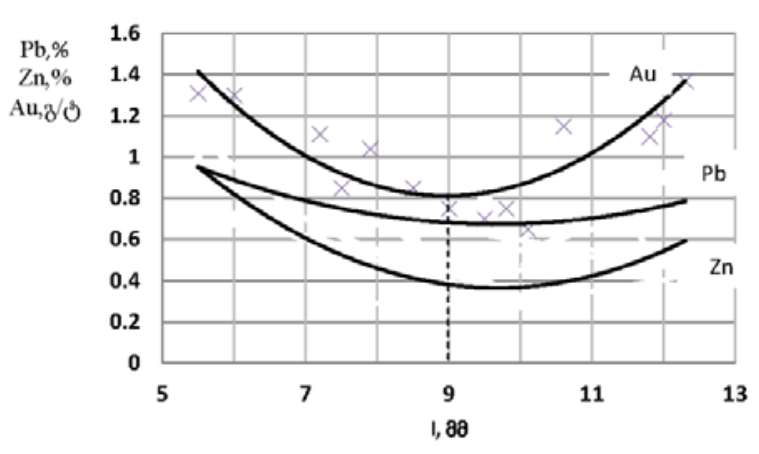

เumต. 3

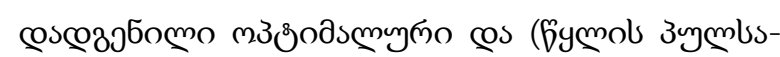

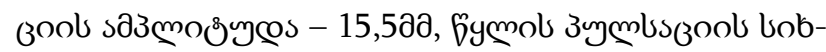

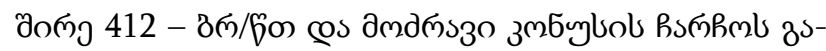

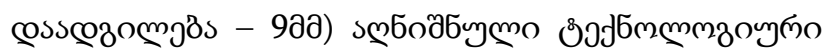

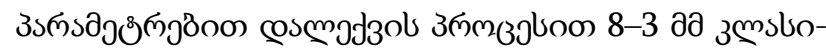

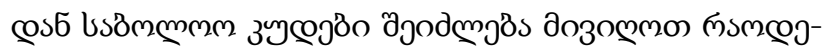

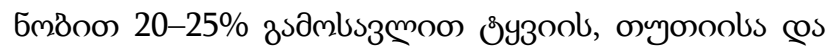

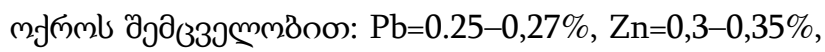
$\mathrm{Au}=0,3-0,5 z / \mathcal{\jmath}$.

\section{costs $336 s$}

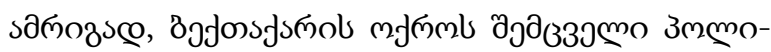

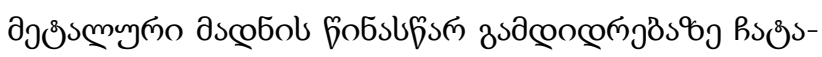

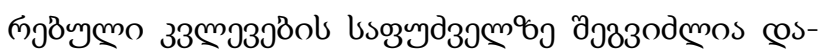

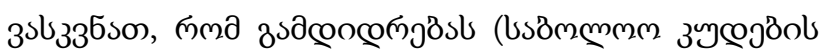

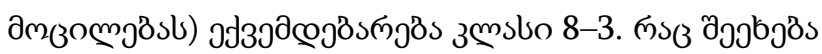

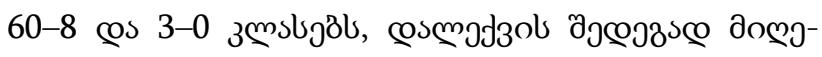

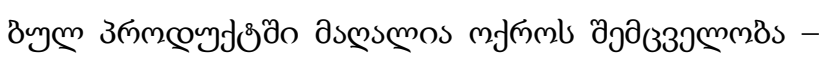

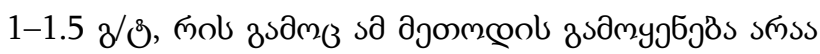

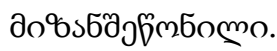

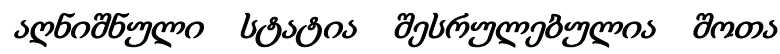

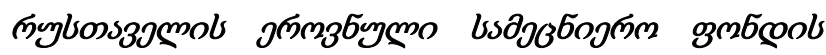

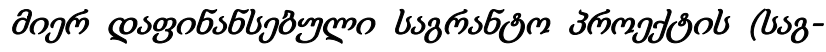

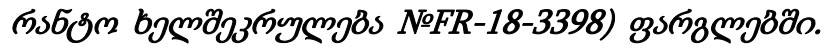

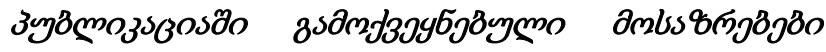

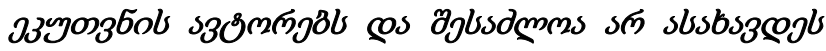

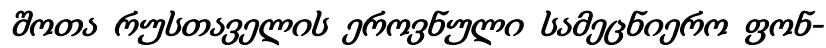
sool ajbjcoygmjojal.

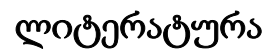

1. Gamtsemlidze M., Enageli R., Rukhadze T. et al. Method for diaphragm jig autocontrol. National Intellectual Property Center of Georgia "SAKPATENTI". GE U 1962, 03.12.2018. (in Georgian).

2. Tarasov A.V., Bocharov V.A. Combined technologies of nonferrous metallurgy. Moscow. Institute Gintsvetmet. 2001. (in Russian).

3. Tarasov A.V., Paretskii V. M. Newest technologies of "Gintsvetmet" in the field of nonferrous metals production and opportunities of their usage in the world. Proceedings of conference metallurgy East-West'97. High Tatras. 1997, 32-36 pp. (in Russian). 
UDC 622.765(075), 625.75.4

SCOPUS CODE 2201

\title{
Study of a pre-enrichment technology for gold-containing polymetallic ores
}

\author{
Demur Talakhadze Department of Mining Technology, Georgian Technical University, 75 M. Kostava str, \\ 0160 Tbilisi, Georgia \\ E-mail: d.talaxadze@gtu.ge \\ Mikheil Gamtsemlidze G.Tsulukidze Mining Institute, 7 E. Mindeli str, 0186 Tbilisi, Georgia \\ E-mail: omar_gamtsemlidze@yahoo.com \\ Roin Enageli Department of Mining Technology, Georgian Technical University, 75 M. Kostava str, \\ 0160 Tbilisi, Georgia \\ E-mail: omar_gamtsemlidze@yahoo.com
}

\section{Reviewers:}

A. Abshilava, Professor, Faculty of Mining and Geology, GTU

E-mail: a.abshilava@gtu.ge

Z. Arabidze, Emeritus, Faculty of Mining and Geology, GTU

E-mail: z.arabidze@gtu.ge

\begin{abstract}
The article presents the results of research of the gold-containing polymetallic ores pre-enrichment technology. The samples were taken from gold-containing polymetallic ores of the Bektakari deposit. In the sample the gold content was $5.61 \mathrm{~g} / \mathrm{t}$, the lead $-1.65 \%$ and the zinc $-2.43 \%$. The material was crushed into $+8 \mathrm{~mm}, 8-3$ $\mathrm{mm}$ and 3-0 $\mathrm{mm}$ classes for large-grain pre-enrichment in order to pre-separate the cleaner tailings. The $+8 \mathrm{~mm}$ class was enriched in a heavy medium (fractional analysis) with a separating density of $2870 \mathrm{~kg} / \mathrm{m} 3$, and the $8-3$ mm class - on a modernized jigging machine. Tests have shown that zinc and lead contents in the tails obtained during the enrichment of $+8 \mathrm{~mm}$ class in a hard medium are minimal, but gold content is high and reaches 1.5-1.7 $\mathrm{g} / \mathrm{t}$. With this in mind, the pre-enrichment of the $+8 \mathrm{~mm}$ class is not recommended. In the tails obtained in the result of jigging of 8-3mm class on a modernized jigging machine, the lead, zinc and gold contents are low, and reach $\mathrm{Pb}=0.24 \%, \mathrm{Zn}=0.32 \%$ and $\mathrm{Au}=0.4 \mathrm{~g} / \mathrm{t}$, respectively. Thus, based on pre-enrichment studies, it was found that: 1 . Pre-enrichment of the $+8 \mathrm{~mm}$ class is not recommended due to the high content of gold in the tails; 2 . By enrichment of the $8-3 \mathrm{~mm}$ class on a modernized jigging machine, $22.3 \%$ of the cleaner tailings with a low grade can be separated.
\end{abstract}

Key words: Analysis; Bektakari deposit; enrichability; gold-containing; jigging process; optimal parameters; ore; tails.

ISSN 1512-0996

www.shromebi.gtu.ge 


\title{
UDC 622.765(075), 625.75.4 \\ SCOPUS CODE 2201
}

\section{Исследование технологии предварительного обогащения золотосодержащих полиметаллических руд}

\author{
Демур Талахадзе Департамент горных технологий, Грузинский технический университет, Грузия, \\ 0160, Тбилиси, ул. М. Костава, 75 \\ E-mail: d.talaxadze@gtu.ge \\ Михаил Гамцемлидзе Горный институт им. Г. Цулукидзе, Грузия, 0186, Тбилиси, ул. Миндели, 7 \\ E-mail: omar_gamtsemlidze@yahoo.com \\ Роин Энагели Департамент горных технологий, Грузинский технический университет, Грузия, \\ 0160, Тбилиси, ул. М. Костава, 75 \\ E-mail: enageli@mail.ru
}

\section{Рецензенты:}
А. Абшилава, профессор горно-геологического факультета ГТУ
E-mail: a.abshilava@gtu.ge
3. Арабидзе, профессор эмеритус горно-геологического факультета ГТУ
E-mail: z.arabidze@gtu.ge

Аннотация. В статье приведены результаты исследований технологии предварительного обогащения золотосодержащих полиметаллических руд. Для исследований взяты пробы из золотосодержащих полиметаллических руд Бектакарского месторождения. В пробе содержание золота было 5,61 гр/т, свинца 1,65\%, цинка $2,43 \%$. Для предварительного обогащения в крупнокусковом виде (целью которого являлось предварительное выделение окончательных хвостов) проба руды измельчена и разделена на классы +8мм, 8-3мм и 3-0мм. Класс крупностью +8мм обогащен в тяжелой среде (фракционный анализ) с плотностью разделения 2870 кг/м³ а класс крупностью 8-3мм обогащен на модернизированной отсадочной машине. На основе данных опытов установлено, что при обогащении в тяжелой среде класса крупности +8 мм, в полученных хвостах содержание цинка и свинца минимальны, а содержание золота высокое и достигает 1,5-1,7 гр/т. Поэтому предварительное обогащение класса крупностью +8 мм не рекомендуется. В результате обогащения класса 8-3 мм на модернизированной отсадочной машине содержание свинца, цинка и золота низкое и соответственно составляет $\mathrm{Pb}=0,24 \%, \mathrm{Zn}=0,32 \%$ и $\mathrm{Au}=0,4$ гр/т. Таким образом, на основе проведенных исследований установлено: 1 . Из-за высокого содержания золота в хвостах предварительное обогащение класса крупности +8 мм не рекомендуется. 2. При обогащении класса крупностью 8-3 мм на модернизированной отсадочной машине возможно выделение 22,3\% окончательных хвостов с минимальным содержанием ценных компонентов.

Ключевые слова: анализ; бектакарское месторождение; руда; золотосодержащий; обогатимость; процесс отсадки; оптимальные параметры; хвосты. 